\title{
Heavy vs Light Load Single-Joint Exercise Performance with Different Rest Intervals
}

\author{
by \\ Gilmar Weber Senna ${ }^{1,2,3}$, Bernardo Minelli Rodrigues ${ }^{2,3}$, Daniel Sandy2, \\ Estevão Scudese ${ }^{2,3}$, Antonino Bianco ${ }^{4}$, Estélio Henrique Martin Dantas 1,2,3
}

The aim of the study was to compare the effect of three distinct rest period lengths between sets of upper body single-joint exercise with different load zones and volume designed for either endurance or hypertrophy $(50 \%$ or $80 \%$ of 1-RM). Sixteen trained men (20.75 \pm 2.54 years; $76.35 \pm 5.03 \mathrm{~kg} ; 176.75 \pm 3.33 \mathrm{~cm}, 24.53 \pm 1.47 \mathrm{~kg} / \mathrm{m} 2)$ performed a test and retest of 1-RM on non-consecutive days. Forty-eight hours after load testing, the participants were randomly assigned to six sessions consisting of four sets of the triceps pull-down, combining different intensities with distinct rest periods between sets. The shorter 1 minute rest promoted a significant reduction in the total repetition number compared to 3 minute rest for both workloads. There was a difference between 3 and 5 minute conditions for the $50 \%$ of 1-RM that did not occur for the $80 \%$ of 1-RM condition. Both intensities presented significant interaction values for the rest conditions vs. each set $(50 \% p=0.0001 ; 80 \% p=0.0001)$. Additionally, significant values were found for the main effect of the performance of subsequent sets $(50 \% p=0.003 ; 80 \% p=0.001)$ and rest conditions $(50 \% p=0.0001$; $80 \% p=0.0001)$. In conclusion, for heavier loads (80\%) to fatigue, longer rest of 3 to 5 minutes seems to allow for better recovery between sets and thus, promotes a greater volume. However, when training with lighter loads (50\%), the magnitude of the rest seems to directly affect the performance of subsequent sets, and also presents a correlation with total volume achieved for the upper body single-joint exercise scheme.

Key words: muscular strength, weight lifting, physical fitness, health promotion.

\section{Introduction}

The rest period between sets has become nowadays an important resistance training variable to be considered within programs (de Salles et al., 2009; Paoli and Bianco, 2015; Pescatello, 2014; Villanueva et al., 2015). It has been demonstrated that its manipulation could trigger distinct neuromuscular (Senna et al., 2011, 2012, 2016), endocrine (Gonzalez et al., 2015; Rahimi et al., 2010), cardiorespiratory (Ratamess et al., 2007) and inflammatory responses
(Rodrigues et al., 2010; Zajac et al., 2015). A number of studies have been carried out in order to refine the time-efficiency of a given workout while maintaining a high level of performance (de Salles et al., 2010; Senna et al., 2016; Willardson and Burkett, 2005, 2006b, 2008). Those new findings might help professionals optimize the physiological adaptation process to the distinct individual goals such as strength, power, hypertrophy or even muscular endurance.

\footnotetext{
1 - Nursing and Biosciences Post-Graduation Program (PPgEnfBio) - Doctorate of Federal University of State of Rio de Janeiro (UNIRIO) - RJ - Brazil..

2 - Laboratory of Biosciences and Human Movement (LABIMH) - Federal University of State of Rio de Janeiro (UNIRIO) - Rio de Janeiro - RJ-Brazil.

3 - Laboratory of Biosciences and Human Movement (LABIMH) - Tiradentes University (UNIT) - Aracajú - SE - Brazil.

4 - Sport and Exercise Science Research Unit - Università degli Studi di Palermo - Palermo - Italy.
} 
In the Position Stand on Progression Models in Resistance Training, the American College of Sports and Medicine (ACSM, 2009) recommends 2 to 3 minute rest intervals between sets for multi-joint (core) exercises and around 1 to 2 minute rest for the single-joint (assistance) exercises. Recently, several authors have shown that different rest period lengths promote distinct training volumes for multi and single-joint exercises at various load intensities (e.g. 3 RM and $10 \mathrm{RM}$ ) and these findings appear to be objective dependent on practical application purposes (Senna et al., 2009, 2011, 2012, 2015, 2016).

For instance, Senna et al. (2011, 2012, 2015, 2016) have described that regardless of the exercise modality (multi or single-joint), rest interval length may determine the number of repetitions performed during subsequent sets. This phenomenon affects the total number of repetitions performed for a targeted exercise (volume) and might influence training goals according to the amount of recovery allowed between sets and subjects' characteristics. Despite similar responses found in performance (volume), the exercise selection (multi or single-joint) could promote significant and distinct metabolic changes (in particular blood lactate elevations). Senna et al. (2012) observed a post-workout blood lactate increase for both types of exercises, however, with a less pronounced increase in blood lactate for the single-joint exercise compared to the multi-joint. Interestingly, the rate of perceived exertion (RPE) was elevated for both conditions and the higher values were more sensitive to the shorter 1 minute recovery. More recently, Senna et al. (2016) observed the performance outcomes for near-maximal loads (3 $\mathrm{RM}$ ) and found a superior performance recovery for the single-joint exercise for the 2 minute rest length compared to the multi-joint exercise. Additionally, when analyzing RPE data between distinct exercises (multi and single-joint) for the same intensity ( $3 \mathrm{RM}$ ), lower scores of the RPE were observed for the single-joint (assistance) exercise in various rest conditions.

However, investigation regarding multi and single-joint exercises for light load zones and high volume to fatigue seems very limited. Wilardson and Burkett (2006a) showed that for multi-joint exercises, independently of the intensity range $(50 \%$ or $80 \%$ of $1-\mathrm{RM})$, the 3 minute rest length seemed to be sufficient to obtain a higher total number of repetitions. More specifically, when examining single-joint exercise recommendation, there is no consensus on the optimal recovery length for the single-joint exercise modality and little is known about its interactions with distinct load designs and rest protocols. This type of exercise is normally implemented as a complementary part of a training regimen. For this reason, this lack of evidence and carelessness observed in the scientific literature might impair the optimal prescription for specific purposes such as the development of a specific sportive task that depends on the enhancement of a single-joint movement. Therefore, the aim of the present investigation was to describe the interactions of various rest periods combined with distinct intensities (50\% and $80 \%$ of $1-\mathrm{RM})$ and analyze the RPE during each set for a single-joint exercise.

\section{Methods}

\section{Participants}

Sixteen trained men $(20.75 \pm 2.54$ years; $76.35 \pm 5.03 \mathrm{~kg} ; 176.75 \pm 3.33 \mathrm{~cm}, 24.53 \pm 1.47$ $\left.\mathrm{kg} / \mathrm{m}^{2}\right)$, with at least one year of resistance exercise experience were invited to participate in the experiment. In order to standardize the participants selection, the following inclusion criteria were implemented: a) subjects should present a training frequency of at least four times per week, around one hour of training per day, and with rest periods close to 1 and 2 minute between sets; b) participants were not allowed to use any ergogenic aids that would enhance performance; c) subjects should not present any acute or chronic health condition that would interfere with exercise performance; d) participants should not engage in physical activities during the experiment. Before data collection, all subjects responded negatively to the PAR-Questionnaire (Shephard, 1988, 2015). Furthermore, all participants read and signed a consent form, elucidating all experiment procedures according to the declaration of Helsinki. The study had been previously approved by the Castelo Branco University Ethics Committee.

\section{Measures}

Forty-eight hours after the familiarization sessions regarding the exercise procedures and 
maximum repetition techniques, all subjects performed the test and retest sessions separated by 48 to 72 hours intending to obtain the individual 1-RM. The test and retest procedures followed the American College of Sports Medicine recommendations (ACSM, 2009; Ehrman, 2010; Pescatello, 2014) according with which every participant performed a warm-up before a maximum of five trials with at least 5 minute of rest between following attempts (Weir et al., 1994). The greatest load successfully reached for the triceps pull-down (single-joint) exercise on both testing sessions was considered as the 1-RM. The exercise execution pattern followed previously established norms and recommendations (Baechle, 2008). All tests were performed at the same time of day for each individual and the warm-up before testing consisted of two sets of 12 repetitions with $40 \%$ of the usual subjects self-estimated 8 RM load (Scudese et al., 2015). Each subject received a standardized verbal encouragement in order to elicit their maximal effort (McNair, 1996).

The adult OMNI Perceived Exertion Scale for Resistance Exercise (OMNI-RES) was implemented to obtain the RPE values. During the initial familiarization process, in order to achieve a better comprehension of the RPE scores, each subject received the OMNI-RES scale along with proper instructions. Then, they were instructed to give scores after each set of their daily training routines to avoid any error during the experiment. Participants were oriented to rank their values according to their perceived effort, muscle tension, discomfort and experienced fatigue during set performance (Lagally and Robertson, 2006). For this experiment, subjects were instructed to rank their RPE immediately after the completion of each set (Scudese et al., 2015; Senna et al., 2016).

\section{Procedures}

Forty-eight hours after the load tests, the subjects were randomly assigned for six training sessions of the single-joint triceps pull-down combining two intensities of $50 \%$ and $80 \%$ of 1 $\mathrm{RM}$ with three rest period lengths of 1,3 and 5 minutes for four sets, respecting 48 hours of recovery between visits. The repetition number was recorded until subjects were unable to perform any further repetition, reaching concentric failure for each set. The subjects were not allowed to pause between the concentric and eccentric phases or even between repetitions at any time during the experiment (Senna et al., 2009). No attempt was made to control the repetition velocity; however, subjects were required to use a smooth and controlled movement for a standardized range of motion (Senna et al., 2011). Immediately after each set, the participants ranked the RPE value using the OMINI-RES scale (Lagally and Robertson, 2006).

\section{Statistical Analysis}

The alpha value adopted was $p \leq 0.05$ and it was used to establish the significance for every comparison. The intraclass correlation coefficient (ICC) was calculated in order to verify the reliability of the measure between the 1-RM test and retest values. The one-way ANOVA was implemented to identify the differences for the total number of repetitions (sum of the number of repetitions following 4 sets) between the distinct rest periods and intensity protocols. Additionally, the two-way ANOVA was performed in order to highlight the interaction between repetition performance for each set and distinct rest periods and intensities separately. When necessary, the Tukey post-hoc analysis was applied for multiple comparisons. Besides the comparisons described, effect size (ES) calculations were carried out to determine the magnitude of the changes. The ES was calculated by the repetition performance values for each intensity and rest period, considering the thresholds proposed by Cohen (1988). Finally, the Friedman test was applied to analyze differences between the RPE values for both rest periods and intensities. When necessary, the Dunnet post-hoc was utilized for multiple comparisons. All statistics calculations were performed using SPSS 21.0 software (IBM, Inc).

\section{Results}

The ICC data showed an excellent correlation $(p=0.99)$ between the test and retest of 1-RM for the triceps pull-down exercise. For the total number of repetitions completed for the lighter $50 \%$ of 1-RM intensity, the one-way ANOVA showed significant decreases when comparing 1 to 3 minute recovery $(p=0.001), 1$ and 5 minute $(p=0.001)$ and 3 to 5 minute $(p=$ 0.005 ) rest periods between sets.

For the heavier $80 \%$ of $1-\mathrm{RM}$, the one-way ANOVA analysis demonstrated significant 
reductions in the total number of repetitions when 1 to 3 minute rest periods $(p=0.001)$ and 1 to 5 minute rest periods were compared $(p=0.001)$. No differences were observed between 3 and 5 minute rest intervals $(p>0.900)$. The total number of repetition data is presented in Table 1.

Both intensities $(50 \%$ and $80 \%$ of $1-\mathrm{RM})$ presented significant interactions for the rest conditions and repetition performance for each set ( $50 \%$ of $1-\mathrm{RM} p=0.0001 ; 80 \%$ of $1-\mathrm{RM} p=0.0001$ ). There were significant decreases in repetition performance for subsequent sets for each rest protocol $(50 \%$ of $1-\mathrm{RM} p=0.003 ; 80 \%$ of $1-\mathrm{RM} p=$ $0.001)$, as well as between rest periods for each set at both intensities ( $50 \%$ of $1-\mathrm{RM} p=0.0001 ; 80 \%$ of 1-RM $p=0.0001$ ).

Progressive performance reductions were observed for all rest protocols (1, 3 and $5 \mathrm{~min})$ starting as early as the second set. Those reductions were much more pronounced with the shorter 1 minute rest period. For the lighter 50\% of $1-\mathrm{RM}$, the shorter 1 minute recovery elicited important reductions from the second set when compared to the longer 3 and 5 minute rest periods. However, this performance impairment appeared with distinct patterns for the heavier $80 \%$ of the 1-RM load. In fact, major decreases were found between the 1 compared to 3 and 5 minute protocol in the third set (Figures 1-4).

The ES calculation indicated that decreases in repetition performance of large magnitude occurred for both intensities and all rest protocols. The ES data are presented in Table 2.

Both intensities triggered elevated scores of the RPE for all rest periods investigated at the third and fourth sets. However, there was an early increase observed for the longer rest periods (3 and $5 \mathrm{~min}$ ) and after the second set for $50 \%$ of 1 RM (Table 3).

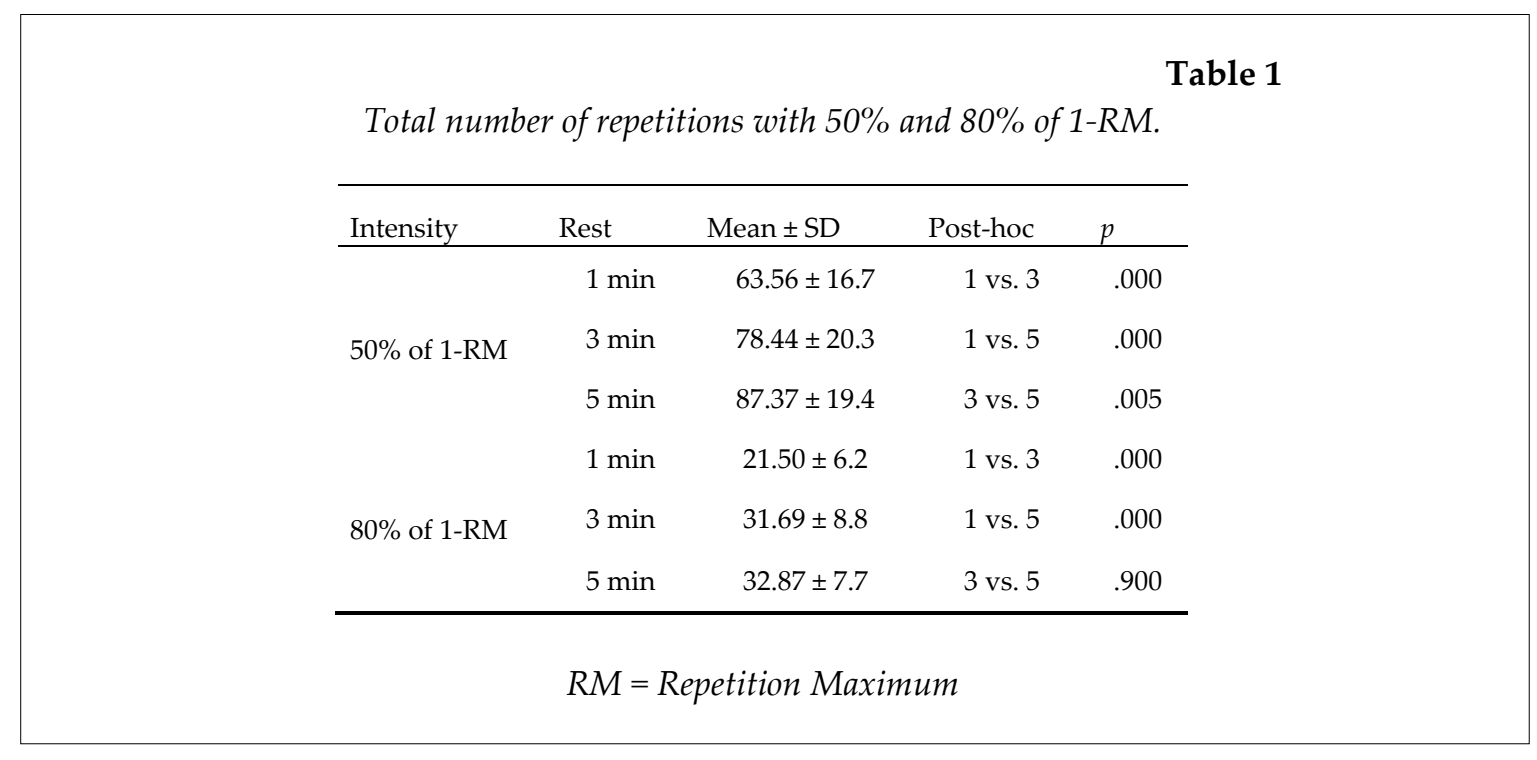

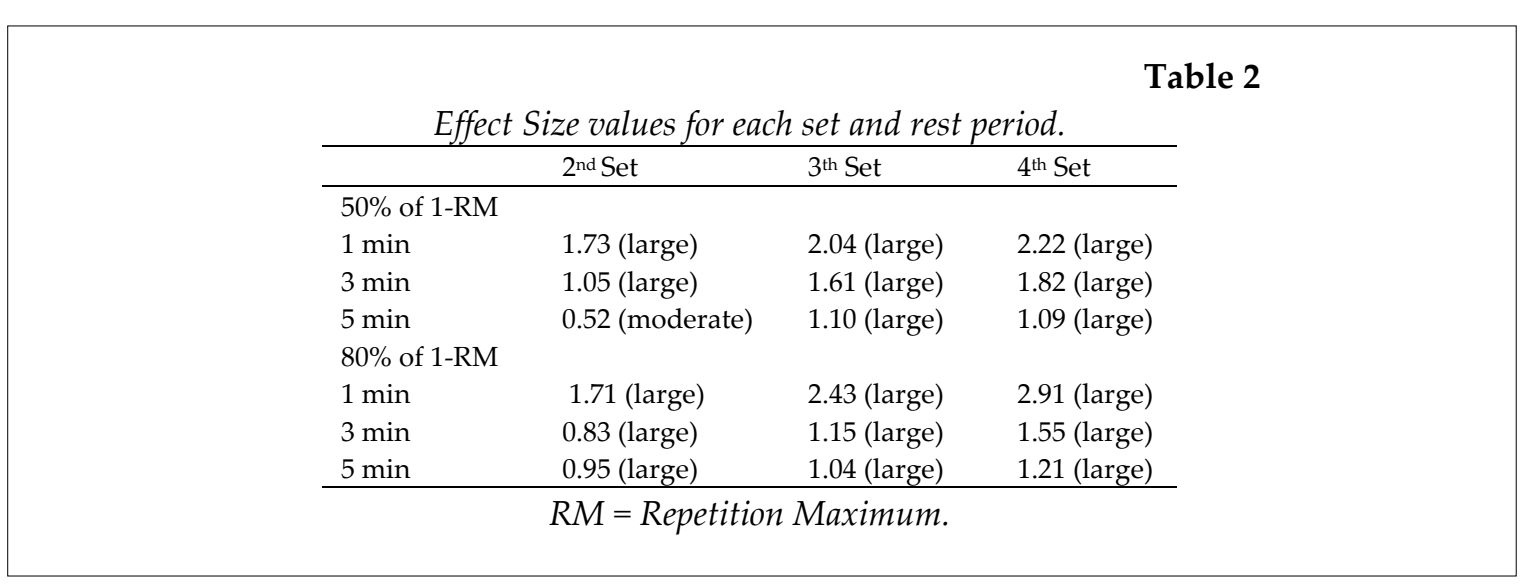


Table 3

\begin{tabular}{|c|c|c|c|c|}
\hline & $1^{\text {st }}$ Set & $2^{\text {nd }}$ Set & $3^{\text {th }}$ Set & $4^{\text {th }}$ Set \\
\hline \multicolumn{5}{|c|}{$50 \%$ of $1-\mathrm{RM}$} \\
\hline $1 \mathrm{~min}$ & 7 & 8 & $9.5^{*}$ & $10^{*}+$ \\
\hline $3 \mathrm{~min}$ & 8 & $9^{* a}$ & $10^{*}$ & $10^{*}+$ \\
\hline $5 \mathrm{~min}$ & 8 & $9^{* a}$ & $9.5^{*}$ & $10^{*}$ \\
\hline \multicolumn{5}{|c|}{$80 \%$ of $1-\mathrm{RM}$} \\
\hline $1 \mathrm{~min}$ & 8 & 9 & $10^{*}$ & $10^{*}+$ \\
\hline $3 \mathrm{~min}$ & 7 & 8 & $10^{*}$ & $10^{*} \dagger$ \\
\hline $5 \min$ & 8 & 8 & $9^{*}+$ & $10^{*}+$ \\
\hline
\end{tabular}

RPE $=$ Rate of Perceived Effort; RM = Repetition Maximum.

* Significant difference to the $1^{\text {st }}$ set.

+ Significant difference to the $2^{\text {nd }}$ set.

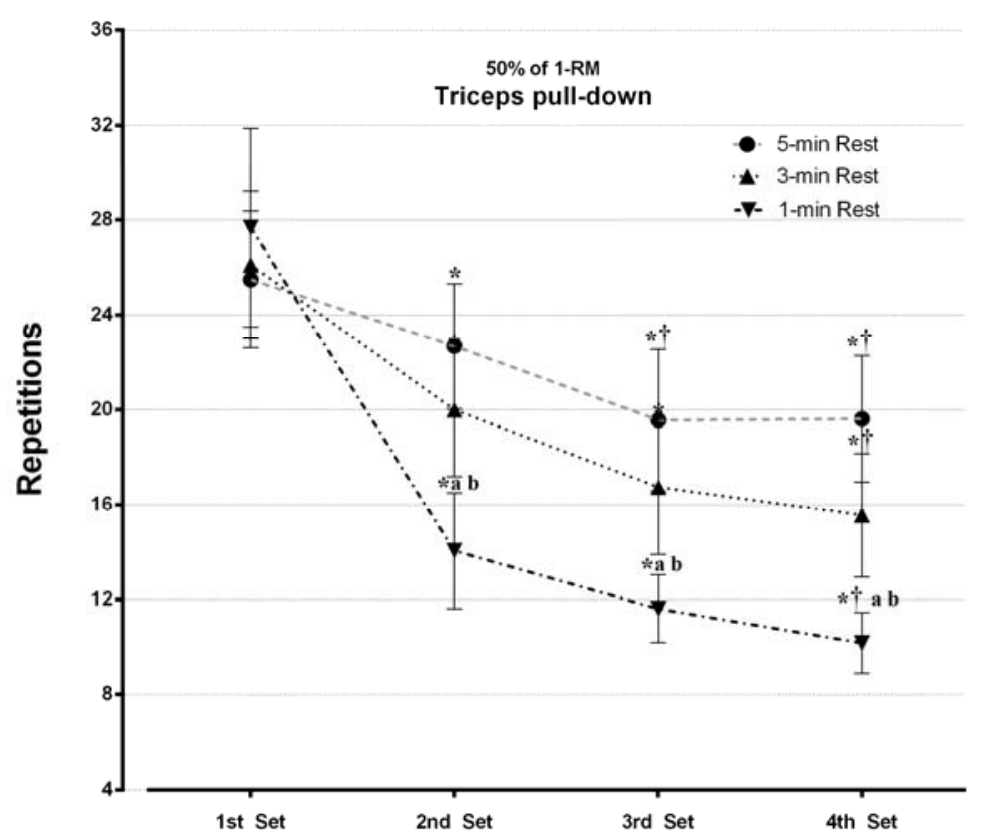

Figure 1

Repetition number for each set to failure with 50\% of 1-RM (Data are mean $\pm S D)$

* Significant difference to the $1^{\text {st }}$ set.

+ Significant difference to the $2^{\text {nd }}$ set.

a Significant difference to 3 minutes of rest.

${ }^{b}$ Significant difference to 5 minutes of rest. 


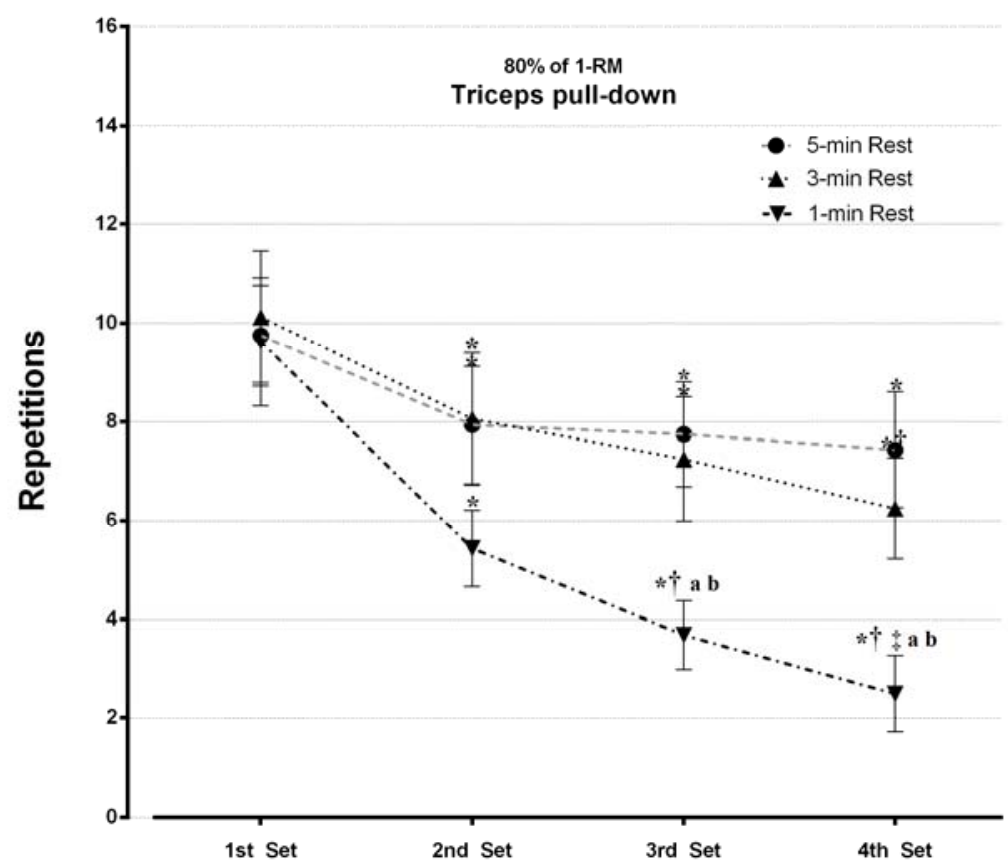

Figure 2

Repetition number for each set to failure with $80 \%$ of 1-RM (Data are mean $\pm S D$ )

* Significant difference to the $1^{\text {st }}$ set. + Significant difference to the $2^{\text {nd }}$ set.

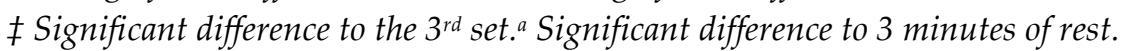

${ }^{b}$ Significant difference to 5 minutes of rest.

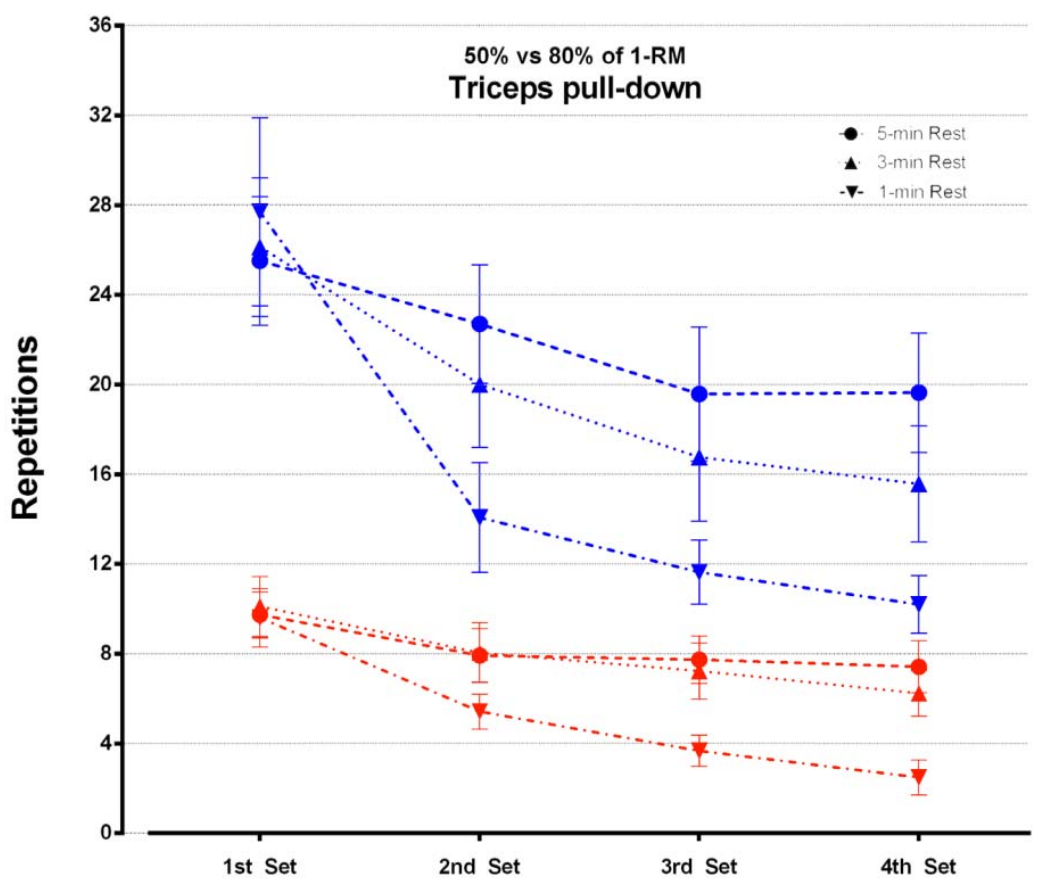

Figure 3

$50 \%$ vs $80 \%$ of the 1-RM triceps pull-down. The $50 \%$ load is reported in blue color while the $80 \%$ load in red color (Data are mean $\pm S D$ ) 




\section{Discussion}

Similar reduction patterns in repetition performance were observed for both intensities ( $50 \%$ and $80 \%$ of $1-\mathrm{RM}$ ) in four consecutive sets for all rest protocols (Figures 1 and 2 and Table 2). When applying the shorter recovery length of 1 minute between sets, a decreased number of repetitions were performed for both lighter $(50 \%$ of $1-\mathrm{RM})$ and heavier loads ( $80 \%$ of $1-\mathrm{RM})$ compared to the longer rest intervals investigated ( 3 and $5 \mathrm{~min}$ ). Performance impairment was observed early in the second set for lighter loads ( $50 \%$ of $1-\mathrm{RM})$ and in the third set for the higher intensity ( $80 \%$ of $1-\mathrm{RM})$. Additionally, progressive reductions were found for the total number of repetitions with shorter rest compared to the longer recovery for the lower intensity $(5>3>1$ $\min )$. For the heavier protocol $(80 \%$ of $1-\mathrm{RM})$, the shorter 1 minute recovery promoted great decreases in the total repetition number compared to the longer 3 and 5 minute rest intervals. However, no differences were found between 3 and 5 minute rest protocols.

In summary, these findings suggest that shorter rest periods $(1 \mathrm{~min})$ for lower intensity regimes such as $50 \%$ of $1-\mathrm{RM}$ to failure, seem to negatively affect the performance of a single-joint exercise. Our results are in accordance with the current recommendation for strength training prescription, suggesting that different rest intervals between sets will promote distinct neuromuscular responses. The performance impairment found is more noticeable when applying shorter rest in comparison to the longer rest conditions.

In addition, for a single-joint exercise, differences between distinct intensities $(50 \%$ and $80 \%$ of 1-RM) became evident around the 3 minute rest range. These findings could help 
elaborate recommendations regarding enhancement in strength, endurance or even hypertrophy for single-joint exercises performed to concentric failure.

The results of our experiment seem to corroborate partially those of Willardson and Burkett (2006a) who analyzed the effects of three different rest periods between sets (1,2 and $3 \mathrm{~min})$ with different intensities (50\% and $80 \%$ of $1-\mathrm{RM}$ ) for an upper body multi-joint exercise. For instance, it was found that the longer the recovery, the higher number of repetitions were performed $(3>2>1 \mathrm{~min})$. In addition, Senna et al. (2011) investigated the effects of distinct rest period lengths on multi and single-joint exercises involving major pectorals and quadriceps muscles for 10 RM loads. The results indicated that for the BP (upper body multi-joint) a greater number of repetitions were performed for the longer 3 and 5 minute conditions compared to the shorter 1 minute rest, with no differences between 3 and 5 minute rest intervals. For all other exercises (LP, MCF and LE), significant differences were found between each rest condition, with greater volume achieved when analyzing longer rest periods compared to the shorter ones ( $5>3>1 \mathrm{~min})$.

Recently, new evidence regarding the load magnitude and repetition range was shown to be determinant when choosing distinct rest period conditions. For instance, the authors (Senna et al., 2016) compared the repetition performance between multi and single-joint exercises with a near-maximal load ( $3 \mathrm{RM}$ ) for multiple distinct rest protocols and demonstrated that in order to achieve high performance sustainability, the 2 minute rest protocol was already sufficient for the single-joint exercise. However, these results were not reproduced when implementing at lighter loads and a high repetition range. For instance, in this study we showed that major performance impairment was found for the 3 minute compared to 5 minute rest protocol.

The RPE values were used to assess the relative intensity of strength training, as previously suggested (Lagally and Robertson, 2006). Prior studies with intensities of $10 \mathrm{RM}$ (Senna et al., 2009, 2011, 2012, 2015) reported increases in the RPE values along consecutive sets, and these elevations were more evident with shorter $(1 \mathrm{~min})$ compared to longer rest periods ( 3 and $5 \mathrm{~min}$ ). More recently, Senna et al. (2016) found significant increases in RPE values for 1 minute rest from the third set for both multi and single-joint exercises in a $3 \mathrm{RM}$ load scheme. This result suggests greater perceived fatigue attained for the shorter recovery condition compared to longer rest periods, independently of the exercise modality (multi or single-joint). In our results, regardless of the intensity, all rest protocols demonstrated RPE values significantly higher from the third set. Specifically, early at the second set, the longer rest periods of 3 and 5 minutes elicited increases in RPE values for lower intensities $(50 \%$ of $1-\mathrm{RM})$. This outcome was probably due to the capability of achieving a high number of repetitions, until reaching concentric failure, allowed by longer rest periods. This may have influenced the RPE data promoting a greatperceived discomfort sensation.

This experiment brings new knowledge regarding the rest interval manipulation effects on very distinct intensities for a single-joint exercise. However, it is important to note that it has some limitations regarding the type of exercise examined (single-joint exercise), population (trained men) and body part (upper body). Therefore, we strongly recommend that future experiments can be elaborated in order to evaluate different exercises, other load ranges (e.g. 15 to $20 \mathrm{RM}$ ), whole body training sessions, and untrained subjects.

In conclusion, we found that the shorter the rest interval, the more dramatic the performance reduction for each intensity tested (50\% and $80 \%$ of $1-\mathrm{RM})$. However, 3 minute rest intervals seem sufficient to allow a recovery similar to that reached after 5 minute rest protocols. Therefore, for a single-joint exercise, differences between intensities $(50 \%$ and $80 \%$ of 1 $\mathrm{RM}$ ) became evident around the 3rd minute of rest. These findings could help elaborate recommendations regarding enhancement in strength, endurance or even hypertrophy for single-joint exercise schemes performed to concentric failure. 


\section{Acknowledgements}

We express our gratitude to all study participants..

\section{References}

ACSM. American College of Sports Medicine position stand. Progression models in resistance training for healthy adults. Med Sci Sports Exerc, 2009; 41: 687-708

Cohen J. Statistical Power Analysis for the Behavioral Sciences. USA, Lawrence Erlbaum Associates; 1988

De Salles BF, Simao R, Miranda F, Novaes JS, Lemos A, Willardson JM. Rest Interval between Sets in Strength Training. Sports Med, 2009; 39: 765-777

De Salles BF, Simão R, Miranda H, Bottaro M, Fontana F, Willardson J. Strength increases in upper and lower body are larger with longer inter-set rest intervals in trained men. J Sci Med Sport, 2010; 13: 429-433

Ehrman JK, de Joung A, Sanderson B, Swain D, Swank A, Womack C. ACSM's Guidelines for Exercise Testing and Prescription; 2010

Gonzalez AM, Hoffman JR, Townsend JR, Adam RJ, Carleigh HB, Kyle SB, Kayla MB, Adam JW, Gerald TM, Edward HR, David DC, Leonardo PO, Darryn SW, David HF, Jeffrey RS. Intramuscular anabolic signaling and endocrine response following high volume and high intensity resistance exercise protocols in trained men. Physiol Rep, 2015; 3: e12466

Lagally KM, Robertson RJ. Construct validity of the OMNI resistance exercise scale. J Strength Cond Res, 2006; 20: 252-256

Mcnair PJ. Verbal encouragement: Effects on maximum effort voluntary muscle action. Brit J Sport Med, 1996; 30: $243-245$

Paoli A, Bianco A. What Is Fitness Training? Definitions and Implications: A Systematic Review Article. Iran J Public Health, 2015; 44: 602-614

Pescatello LS, Arena R, Riebe D, Thompson PD. ACSM's Guidelines for Exercise Testing and Prescription. 9th Ed, Philadelphia, US, Wolters Kluwer/Lippincott Williams \& Wilkins; 2014.

Rahimi R, Qaderi M, Faraji H, Boroujerdi SS. Effects of very short rest periods on hormonal responses to resistance exercise in men. J Strength Cond Res, 2010a; 24: 1851-1859

Ratamess NA, Falvo MJ, Mangine GT, Hoffman JR, Faigenbaum AD, Kang J. The effect of rest interval length on metabolic responses to the bench press exercise. Eur J Appl Physiol, 2007; 100: 1-17

Rodrigues BM, Dantas E, De Salles BF, Miranda H, Koch AJ, Willardson JM, Simão R. Creatine kinase and lactate dehydrogenase responses after upper-body resistance exercise with different rest intervals. $J$ Strength Cond Res, 2010; 24: 1657-1662

Scudese E, Willardson JM, Simao R, Senna G, de Salles BF, Miranda H. The effect of rest interval length on repetition consistency and perceived exertion during near maximal loaded bench press sets. J Strength Cond Res, 2015; 29: 3079-83

Senna G, Salles BF, Prestes J, Mello RA, Simão R. Influence of two different rest interval lengths in resistance training sessions for upper and lower body. J Sport Sci Med, 2009; 8: 197-202

Senna G, Scudese E, Carneiro F, Torres J, Queiroz C, Dantas E. Multi-joint and single-joint exercise performance and perceived exertion with several different recoveries. J Exercise Phys Online, 2015; 18: 91-100

Senna G, Willardson JM, De Salles BF, Scudese E, Carneiro F, Palma A, Simão R. The effect of rest interval length on multi and single-joint exercise performance and perceived exertion. J Strength Cond Res, 2011; 25: 3157-62

Senna GW, Figueiredo T, Scudese E, Baffi M, Carneiro F, Moraes E, Miranda H, Simão R. Influence of different rest interval lengths in multi-joint and single-joint exercises on repetition performance, perceived exertion, and blood lactate. J Exercise Phys Online, 2012; 15: 96-106 
Senna GW, Willardson JM, Scudese E, Simão R, Queiroz C, Avelar R, Dantas EHM. Effect of Different Interset Rest Intervals on Performance of Single and Multijoint Exercises With Near-Maximal Loads. J Strength Cond Res, 2016; 30: 710-716

Shephard RJ. PAR-Q, Canadian Home Fitness Test and exercise screening alternatives. Sports Med, 1988; 5: 185-195

Shephard RJ. Qualified Fitness and Exercise as Professionals and Exercise Prescription: Evolution of the PAR-Q and Canadian Aerobic Fitness Test. J Phys Act Health, 2015; 12: 454-461

Baechle TR, Earle RW. Essentials of strength training and conditioning, Champaign, IL 61825-5076, Human Kinetics; 2008

Villanueva MG, Lane CJ, Schroeder ET. Short rest interval lengths between sets optimally enhance body composition and performance with 8 weeks of strength resistance training in older men. Eur J Appl Physiol, 2015; 115: 295-308

Weir JP, Wagner LL, Housh TJ. The effect of rest interval length on repeated maximal bench presses. J Strength Cond Res, 1994; 8: 58-60

Willardson JM, Burkett LN. A comparison of 3 different rest intervals on the exercise volume completed during a workout. J Strength Cond Res, 2005; 19: 23-6

Willardson JM, Burkett LN. The effect of rest interval length on bench press performance with heavy vs. light loads. J Strength Cond Res, 2006a; 20: 396-9

Willardson JM, Burkett LN. The effect of rest interval length on the sustainability of squat and bench press repetitions. J Strength Cond Res, 2006b; 20: 400-3

Willardson JM, Burkett LN. The effect of different rest intervals between sets on volume components and strength gains. J Strength Cond Res, 2008; 22: 146-152

Zajac A, Chalimoniuk M, Maszczyk A, Golas A, Lngfort J. Central and Peripheral Fatigue During Resistance Exercise - A Critical Review. J Hum Kinet, 2015; 49: 159-69

\section{Corresponding author:}

\section{Antonino Bianco, PhD, Assistant Professor}

Sport and Exercise Sciences Research Unit

University of Palermo

Via Giovanni Pascoli 6 - 90144

Palermo, Italy

Tel. 003909123896910

Fax. 003909123860894 